doi:10.1016/j.jclinepi.2006.03.010

Copyright (c) 2006 Elsevier Inc. All rights reserved.

\title{
Disparities in stroke preventive care in general practice did not explain socioeconomic disparities in stroke
}

\section{Avendano ${ }^{a, b}, *$, H.C. Boshuizen ${ }^{b}$, F.G. Schellevis ${ }^{c}$, J.P. Mackenbach ${ }^{a}$, F.J. Van Lenthe ${ }^{a}$ and G. A.M. Van den Bos ${ }^{d}$}

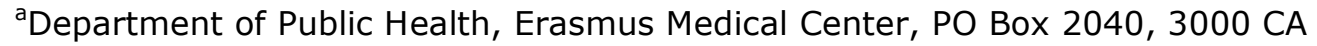
Rotterdam, The Netherlands

${ }^{b}$ National Institute of Public Health and the Environment, Bilthoven, The Netherlands

${ }^{c}$ Netherlands Institute for Health Services Research (Nivel), Utrecht, The Netherlands

${ }^{\mathrm{d}}$ Department of Social Medicine, Academic Medical Center, University of Amsterdam, Amsterdam, The Netherlands

* Corresponding author. Tel.: +31-10-408-7507; fax: +31-10-463-8460.

\section{Abstract \\ Objective}

To assess socioeconomic disparities in stroke incidence and in the quality of preventive care for stroke in the Netherlands.

\section{Study Design and Settings}

A total of 190,664 patients who registered in 96 general practices were followed up for 12 months. Data were collected on diagnoses, referrals, prescriptions, and diagnostic procedures. Hazard ratios (HR) were calculated to assess the association between educational level and stroke incidence. Multilevel logistic regression was used to assess socioeconomic disparities in the quality of preventive care for stroke precursors.

\section{Results}

Lower educational level was associated with higher incidence of stroke in men ( $\mathrm{HR}=1.36,95 \% \mathrm{CI}=1.06-1.74)$ but not in women. Among both men and women, there were socioeconomic disparities in the prevalence of hypertension, hypercholesterolemia, diabetes, angina pectoris, heart failure, and peripheral artery disease. Lower educated hypercholesterolemia patients under medication were less likely to be prescribed statins (odds ratio $=0.62,95 \% \mathrm{CI}=0.42-0.91$ ) . However, for other precursors of stroke, there were no major disparities in the quality of preventive care.

\section{Conclusion}

There are socioeconomic disparities in stroke incidence among men but not among women. Socioeconomic differences in factors such as hypertension and diabetes are likely to contribute to stroke disparities. However, general 
practitioners(GPs) provide care of a similar quality to patients from different socioeconomic groups.

Keywords: Cerebrovascular accident; Social class; Incidence; Prevention and control; Quality of healthcare; Family practice

\section{Introduction}

Stroke is the second leading cause of death and one of the major causes of disability worldwide accounting for 4.3 million deaths every year [1]. A lower socioeconomic status is associated with a higher stroke risk [2], [3], [4], [5], [6], [7], [8] and [9], and can account for more than one third of strokes [5]. Moreover, stroke contributes considerably to socioeconomic differences in life expectancy [8]. Previous research reported that socioeconomic deprivation is associated with a poor management of cardiovascular risk factors such as hypertension [10]. This underlines a large potential for stroke prevention by improving primary care interventions in the lower socioeconomic groups.

European countries have a long tradition of universal healthcare coverage, so that access to care is guaranteed to the whole population. In the Netherlands, general practice care is exceptionally well organized. Almost all individuals in the Dutch population are registered with a general practice, and care is accessible to all through health insurance schemes. Thus, in principle, the quality of care should be equal for all individuals. However, socioeconomic disparities in healthcare utilization persist [11]. As in most European countries, individuals with a lower socioeconomic status use more general practice care, even after adjustment for health need [11] and [12]. Conversely, the higher socioeconomic groups use more specialist care [11] and [12]. It is not known whether there might also be socioeconomic differences in the quality of care. Overall, the mechanisms through which healthcare can contribute to health disparities remain largely unexplored [13].

Since 1989, the Dutch College of General Practitioners has introduced guidelines for the management of specific diagnoses [14]. This includes guidelines to improve the quality of GPs' care for stroke precursors such as hypertension and diabetes mellitus, covering specific aspects of care such as medical prescriptions and diagnostic procedures. Guidelines can refer to general aspects for the prevention of stroke, such as hypertension treatment, or specific aspects for the prevention of ischemic or hemorrhagic stroke only, for example, aspirin prescription for ischemic stroke prevention. Few studies have systematically assessed socioeconomic disparities in preventive care provided by GPs. Research on these disparities may reveal specific aspects of care that can help prevent socioeconomic disparities in stroke occurrence.

This study assesses socioeconomic disparities in stroke incidence, and how these relate to socioeconomic disparities in the quality of preventive care for precursors of stroke (Fig. 1). Previous studies have only focused on disparities in hospital care or have faced some methodological limitations, looking mostly at healthcare utilization [10], [11] and [15]. We applied an innovative approach by developing quality indicators based on evidenced-based guidelines for stroke prevention at general practice. Building upon previous research, we assessed deviations from general practice guidelines relevant for stroke prevention, and incorporated adjustment for patients' health need. We applied multilevel analyses techniques, 
thus taking into account general practice variations in the analyses of socioeconomic disparities in care quality. We used data from the second Dutch national survey, a unique study comprising data on more than 190,000 patients registered with 96 general practices in the Netherlands and followed up for a period of one year. This is the first study to use quality indicators to assess socioeconomic differences in care in general practice and to focus on preventive interventions.

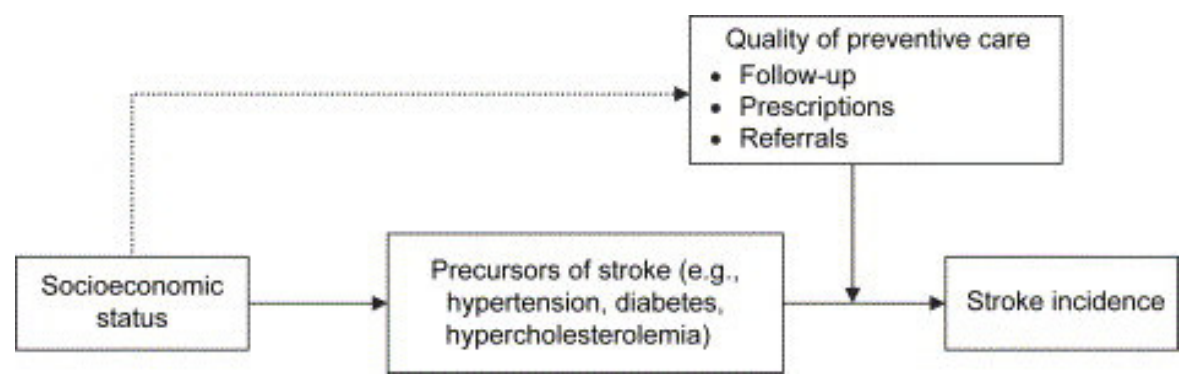

Fig. 1. Conceptual model of the contribution of precursors of stroke and preventive care to socioeconomic differences in stroke incidence.

\section{Methods}

\subsection{Data and subjects}

Within the framework of the second Dutch national survey of general practice, 104 general practices participated in a study of general practice care carried out between May 2000 and April 2002. Details of the study have been described elsewhere [16]. To obtain a representative sample, practices were selected on the basis of three classification criteria: region (north, central, and south), level of urbanization (five categories), and practice type (individual vs. group). GPs from these practices $(n=195)$ were representative of the Dutch GP population in terms of age, gender, residence, and level of urbanization. Similarly, patients were a representative sample of the Dutch population in terms of age, gender, and type of health insurance.

To obtain information on demographic variables and socioeconomic status, a baseline survey was carried out among all registered patients. A response rate of $76.5 \%$ was obtained. Data on patients' contacts with the GP were collected during a follow-up period of 12 months. For each contact, GPs recorded information on diagnoses, referrals, and drug prescriptions. Information was recorded electronically at the practice and subsequently integrated into a central information system. Contacts for a single diagnosis were grouped into a disease episode. Additionally, data on specific diagnostic procedures performed by the GP were recorded for a period of 6 weeks, including blood pressure and weight measurements.

Educational level was used as an indicator of socioeconomic status, because it can be applied in a comparable way for both men and women. Furthermore, as opposed to other indicators such as occupational class, educational level can be applied to both the economically active and inactive population [8] and [17]. Previous studies have also shown that educational level is consistently related to stroke [4] and [8]. Individuals were asked about the highest level of education they had completed. Reported levels were recoded into three categories: low (no schooling or solely elementary education), middle (secondary school), and high (postsecondary education). 
To assess the quality of preventive care, eight quality indicators were constructed on the basis of national guidelines provided by the Dutch College of General Practitioners [14] (Table 1). Indicators 1-4 were developed by the Centre for Quality of Care Research (WOK) through an iterative consensus procedure, and were defined in terms of expected health outcomes and the prevention of unnecessary interventions [18] and [19]. Indicators 5-8 were constructed by the investigators on the basis of practice guidelines for blood pressure measurements, specialist referrals, and diabetes control [14] and [20]. Each indicator comprised a numerator and a denominator [18]. Numerators denoted the number of patients who received a specific type of care according to the guidelines. Denominators denoted the target population that, according to the guidelines, should in principle receive a specific type of care. This innovative approach allows the assessment of quality of care by measuring adherence to guidelines and specifying the target group. Most quality of care indicators referred to guidelines for the prevention of all stroke subtypes (ischemic stroke, and intracerebral and subarachnoid hemorrhage). The only exceptions were indicators 2 and 3 regarding the prescription for statins and aspirin, which are directly relevant for the prevention of ischemic stroke only [20]. 
Table 1.

Indicators of the quality of care for stroke prevention constructed on the basis of guidelines of the Dutch College of General Practitioners

\begin{tabular}{|c|c|c|}
\hline & \multicolumn{2}{|l|}{ Quality of care indicators } \\
\hline & Indicator (numerator) & Target population (denominator) \\
\hline 1. & Prescription for diuretics (as first step of second-line medication treatment) & $\begin{array}{l}\text { Patients with uncomplicated hypertension under medication } \\
\text { (diuretics, beta-blockers, ACE-inhibitors, or calcium antagonists) }\end{array}$ \\
\hline 2. & Prescription for statins (as second-line medication treatment) & $\begin{array}{l}\text { Patients with hypercholesterolemia under lipid-reducing medication } \\
\text { (relevant for preventing ischemic stroke only) }\end{array}$ \\
\hline 3. & Prescription for aspirin & $\begin{array}{l}\text { Patients with a diagnosis of transient ischemic attack, peripheral artery disease or, angina pectoris } \\
\text { (relevant for preventing ischemic stroke only) }\end{array}$ \\
\hline 4. & Combined prescription for both diuretics and ACE-inhibitors & Heart failure patients with a prescription for ACE-inhibitors \\
\hline 5. & Referral to the specialist (neurologist or cardiologist) & $\begin{array}{l}\text { Transient ischemic attack, angina pectoris, peripheral artery disease, } \\
\text { and patients with heart failure }\end{array}$ \\
\hline 6. & Weight measurement (diabetes) & Diabetes mellitus patients who visited the GP during a 6-week study period \\
\hline 7. & Blood pressure measurement (diabetes) & Diabetes mellitus patients who visited the GP during a 6-week study period \\
\hline 8. & Blood pressure measurement & Patients aged 60 years and over who visited the GP during a 6-week study period \\
\hline
\end{tabular}


Diagnoses by the GP were coded according to the International Classification of Primary Care coding system [21], using the following classification: K90 (stroke), K89 (transient ischemic attack), hypertension (K86, K87), diabetes mellitus (T90), angina pectoris (K74), heart failure (K77), peripheral artery disease (K92), and hypercholesterolemia (T93). Medication prescriptions by the GP were coded according to the Anatomic, Therapeutic, and Chemical (ATC) classification of pharmaceutical drugs [22], using the following classification: diuretics (C03), beta-blockers (C02, C07), ACE inhibitors (C09), calcium antagonists (C08), and cholesterol-lowering medication agents (C10).

\subsection{Methods of analysis}

Eight practices were excluded from the analyses due to problems in data collection and quality. Thus, the base population at risk consisted of 385,461 individuals registered in 96 practices. For the present study, we included only individuals aged 25 years and over, and for whom data on educational level were available, resulting in a final sample of 190,665 subjects.

To assess the effect of educational level on stroke, age-standardized stroke incidence rates were calculated separately for men and women, using the Dutch population of 2000 as the standard [23]. Hazard ratios were calculated using the Cox Proportional Hazard model, incorporating age as the time scale [24]. No information was available on stroke occurrence before the start of the study. Therefore, we included both first and recurrent stroke events that occurred during the study period, applying a Cox Proportional Hazard model for multiple events and adjusting for the dependency of outcomes [25].

Significant practice variation existed in diagnoses and healthcare use patterns. Thus, multilevel analyses techniques were applied to assess the effect of educational level on the prevalence of stroke precursors and preventive care. Firstly, we calculated age-adjusted prevalence rates and odds ratios of stroke precursors according to educational level, using multilevel logistic regression. Secondly, compliance of GPs with practice guidelines was calculated for each quality indicator, adjusting for age. Thirdly, to further adjust for health need, multilevel logistic regression was applied, adjusting for age, self-perceived health, cardiovascular symptoms, specific cardiovascular diseases, diabetes, and other comorbidities. Because patients may have received certain types of care from the specialist, analyses were also adjusted for specialist referrals. Given the smaller size of patient subsamples, odds ratios compared the low educational level group with the middle/high educational level group. These two upper levels were combined to obtain more precise estimates.

Survival analyses were performed using the statistical package SAS version 8.2 (SAS Institute, Cary, NC). Multilevel analyses were conducted using the statistical package MLWIN version 1.02 (Centre for Multilevel Modelling, Institute of Education, London, UK).

\section{Results}

The mean age of registered patients at general practice was 49.5 for men and 50.7 for women. A total of 472 individuals developed stroke over 187,220 personyears. The mean age at stroke diagnosis was 70.9 for men and 76.1 for women. Table 2 presents the number of strokes, person-years, incidence rates, and hazard ratios of stroke according to educational level. Among men, the agestandardized stroke incidence rate steadily increased as educational level decreased, yielding a clear socioeconomic gradient $(P$ for trend $<0.05)$. The 
stroke incidence rate was higher among men with a low educational level than among men in the middle/high educational category ( $\mathrm{HR}=1.36,95 \% \mathrm{CI}=1.06-$ 1.74). Among women, however, the stroke incidence rate did not differ between educational level groups $(H R=1.05,95 \% C I=0.79-1.38)$.

Table 2.

Age-standardized stroke incidence rates and hazard ratios by educational level among men and women aged 25 years and over

\begin{tabular}{|c|c|c|c|c|c|c|}
\hline Sex & $\begin{array}{l}\text { Socioeconomic } \\
\text { status }\end{array}$ & $\begin{array}{l}\text { No. of } \\
\text { strokes }\end{array}$ & $\begin{array}{l}\text { Person- } \\
\text { years }\end{array}$ & $\begin{array}{l}\text { Incidence } \\
\text { rate }\end{array}$ & $\begin{array}{l}\text { Hazard } \\
\text { ratio }\end{array}$ & $95 \%$ CI \\
\hline \multirow[t]{5}{*}{ Men } & \multicolumn{6}{|l|}{ Educational level } \\
\hline & High & 30 & 22434.6 & 218.7 & 1.00 & \\
\hline & Middle & 113 & 52215.7 & 267.6 & 1.22 & $0.82-1.81$ \\
\hline & Low & 110 & 14429.1 & 354.0 & 1.58 & $1.07-2.36^{*}$ \\
\hline & Middle/high vs. low & & & & 1.36 & $1.06-1.74$ \\
\hline \multirow[t]{5}{*}{ Women } & \multicolumn{6}{|l|}{ Educational level } \\
\hline & High & 11 & 17443.2 & 197.1 & 1.00 & \\
\hline & Middle & 82 & 58238.0 & 180.3 & 1.08 & $0.57-2.04$ \\
\hline & Low & 126 & 22459.7 & 189.1 & 1.12 & $0.59-2.14$ \\
\hline & Middle/high vs. low & & & & 1.05 & $0.79-1.38$ \\
\hline
\end{tabular}

$* P$-value for trend effect of educational level $<0.05$.

Table 3 shows the age-adjusted prevalence (per 1,000 ) of precursors of stroke. Men and women with a low educational level had a less favorable risk profile. $A$ low educational level was associated with a higher prevalence of hypertension, hypercholesterolemia, diabetes mellitus, angina pectoris, heart failure, and peripheral artery disease. There was a marked educational level gradient in the prevalence of these factors for both men and women ( $P$ for trend $<0.05$ ). A low educational level was associated with a higher prevalence of transient ischemic attack (TIA) in women, but not in men. Odds ratios in Fig. 2 and Fig. 3 show that men and women with a low educational level consistently had a higher prevalence of precursors of stroke than those with a middle or high educational level. 
Table 3.

Age-adjusted prevalence (per 1,000) of precursors of stroke according to educational level among men and women aged 25 years and over

\begin{tabular}{|l|l|l|l|l|l|l|l|l|}
\hline Stroke precursor & \multicolumn{2}{l}{ Men } & \multicolumn{4}{l}{ Women } \\
\hline & \multicolumn{2}{l}{ Educational level } & \multicolumn{4}{l|}{ Educational level } \\
\hline & High & Middle & Low & Total & High & Middle & Low & Total \\
\hline Hypertension & 77.3 & 84.5 & $87.6 *$ & 82.1 & 97.6 & 114.6 & $127.2^{*}$ & 115.8 \\
\hline Hypercholesterolemia & 28.0 & 33.3 & $34.8^{*}$ & 32.3 & 19.0 & 25.5 & $28.2 *$ & 25.0 \\
\hline Transient ischemic attack & 5.5 & 6.0 & 5.2 & 5.6 & 3.4 & 5.8 & 5.5 & 5.6 \\
\hline Diabetes mellitus & 27.7 & 36.1 & $53.8^{*}$ & 37.9 & 19.9 & 34.0 & $59.4 *$ & 40.9 \\
\hline Angina pectoris & 13.6 & 18.8 & $23.5 *$ & 19.0 & 11.1 & 13.5 & $17.9 *$ & 15.3 \\
\hline Heart failure & 7.2 & 9.8 & $13.2^{*}$ & 10.7 & 7.2 & 9.5 & $13.6 *$ & 11.6 \\
\hline Peripheral artery disease & 3.6 & 6.0 & $7.6 *$ & 5.9 & 1.8 & 3.3 & $4.4 *$ & 3.4 \\
\hline
\end{tabular}

$* P$-value for trend effect of educational level $<0.05$

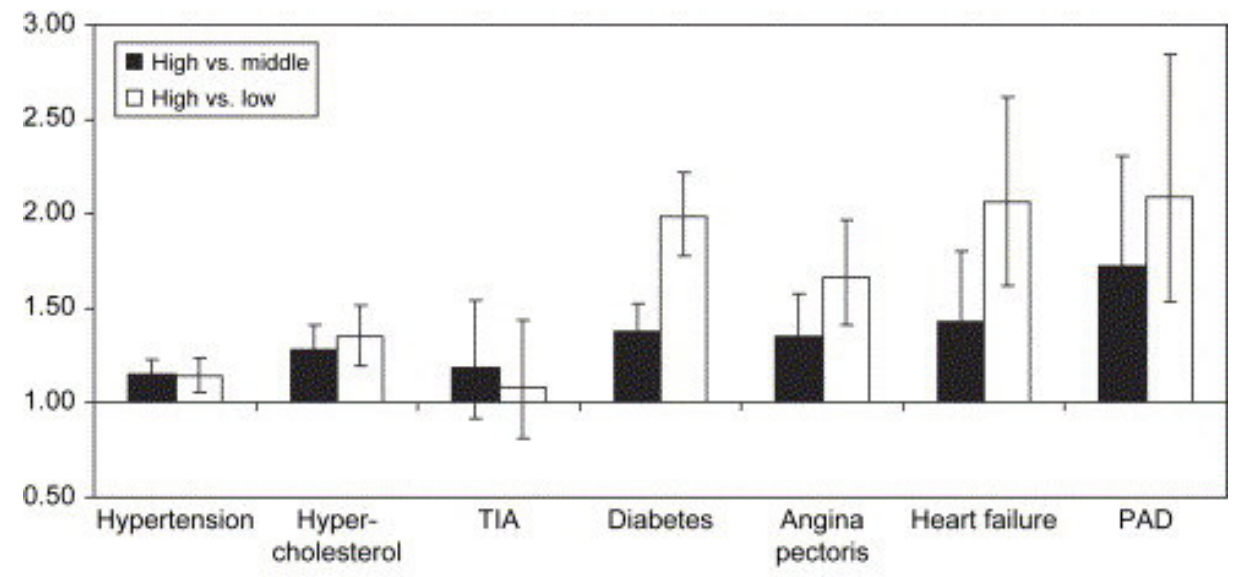

Fig. 2. Age-adjusted odds ratios of precursors of stroke according to educational level for men aged 25 years and above. PAD, peripheral artery disease. 


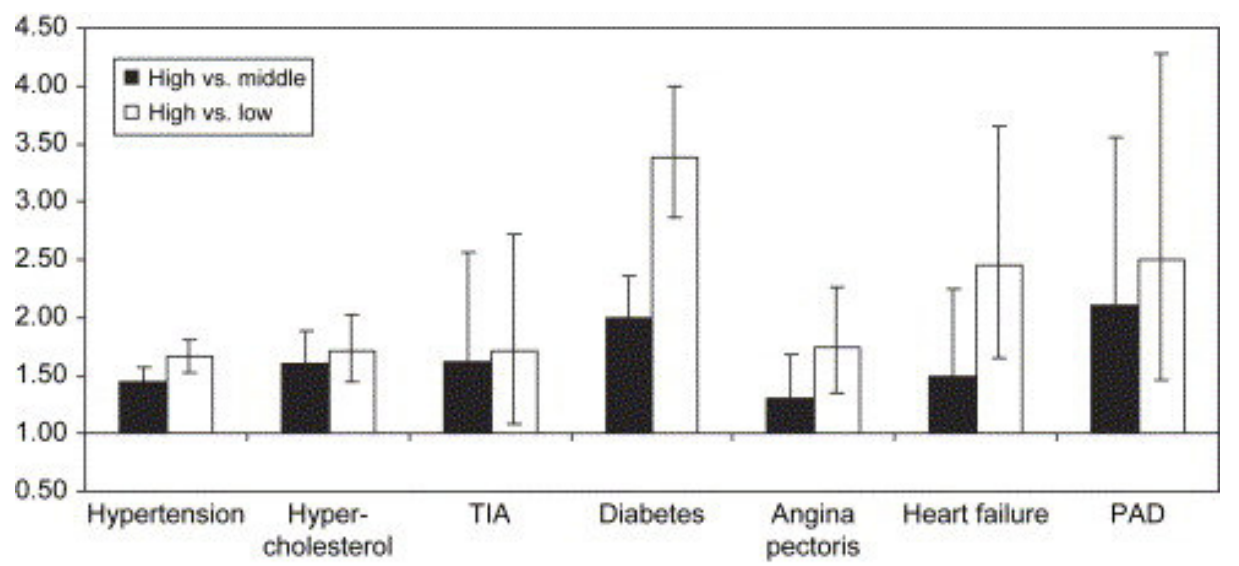

Fig. 3. Age-adjusted odds ratios of precursors of stroke according to educational level for women aged 25 years and above. PAD, peripheral artery disease.

Table 4 shows the profile of healthcare for precursors of stroke, on the basis of eight quality of care indicators. GPs' overall compliance with some guidelines was low: only about one third of men and one half of women with uncomplicated hypertension received diuretics as first step of second-line medication treatment (indicator 1). Approximately half of the patients with a diagnosis of TIA, peripheral artery disease, or angina pectoris had received an aspirin prescription (indicator 3). Similarly, only about two thirds of patients with heart failure had a combined prescription for ACE-inhibitors and diuretics (indicator 4). Nevertheless, the quality of care for patients with a low educational level was similar or better than the quality of care for patients with a higher educational level. A prescription for diuretics as the first step of second-line medication treatment for uncomplicated hypertension (indicator 1 ) was somewhat more common in lower educated patients for both men (OR $=1.22,95 \% \mathrm{CI}=1.05-1.42)$ and women $(\mathrm{OR}=1.10,95 \% \mathrm{CI}=1.00-1.22)$. The patterns of prescription for aspirin (indicator 3 ) and ACE-inhibitors and diuretics combined (indicator 4) did not differ according to educational level. Similarly, cardiovascular patients with a low educational level were referred to the specialist as often as patients from the higher educational categories (indicator 5). Among diabetic patients, blood pressure and weight were measured as frequently in all socioeconomic groups (indicators 6 and 7 ). Individuals aged 60 years and over with a low educational level had their blood pressure measured as frequently as patients with a higher educational level (indicator 8 ). Thus, overall, the quality of care for stroke precursors was of a similar quality for patients with different socioeconomic status. This pattern was observed even after adjusting for health need (Table 4). 
Table 4.

Age-standardized percentages and adjusted odds ratios ${ }^{\mathrm{a}}$ of GPs' compliance with guidelines for stroke prevention among men and women aged 25 years and above

\begin{tabular}{|c|c|c|c|c|c|c|c|}
\hline & \multirow[t]{3}{*}{ Indicator } & \multicolumn{3}{|l|}{ Men } & \multicolumn{3}{|c|}{ Women } \\
\hline & & \multicolumn{3}{|c|}{ Educational level } & \multicolumn{3}{|c|}{ Educational level } \\
\hline & & $\boldsymbol{N}$ & Middle/high & Low & $\boldsymbol{N}$ & Middle/high & Low \\
\hline \multirow[t]{3}{*}{1.} & Diuretics for uncomplicated hypertension patients under medication & 5,274 & $33.1 \%$ & $37.3 \%$ & 9,279 & $46.1 \%$ & $49.3 \%$ \\
\hline & & & 1.00 & 1.22 & & 1.00 & 1.10 \\
\hline & & & & $(1.05-1.42)$ & & & $(1.00-1.22)$ \\
\hline \multirow[t]{3}{*}{2.} & Statins for hypercholesterolemia patients under medication & 2,869 & $97.5 \%$ & $95.4 \%$ & 2,361 & $97.9 \%$ & $95.3 \%$ \\
\hline & & & 1.00 & 0.64 & & 1.00 & 0.60 \\
\hline & & & & $(0.37-1.11)$ & & & $(0.34-1.05)$ \\
\hline \multirow[t]{3}{*}{3.} & Aspirin prescription for $\mathrm{TIA}, \mathrm{PAD},{ }^{\mathrm{b}}$ and angina pectoris patients & 3,207 & $55.5 \%$ & $56.0 \%$ & 2,885 & $51.8 \%$ & $51.6 \%$ \\
\hline & & & 1.00 & 0.97 & & 1.00 & 1.01 \\
\hline & & & & $(0.82-1.15)$ & & & $(0.85-1.21)$ \\
\hline \multirow[t]{2}{*}{4.} & ACE-inhibitors and diuretics prescription combined for heart failure patients & 570 & $64.8 \%$ & $62.7 \%$ & 661 & $66.5 \%$ & $60.1 \%$ \\
\hline & & & 1.00 & 0.97 & & 1.00 & 0.74 \\
\hline
\end{tabular}




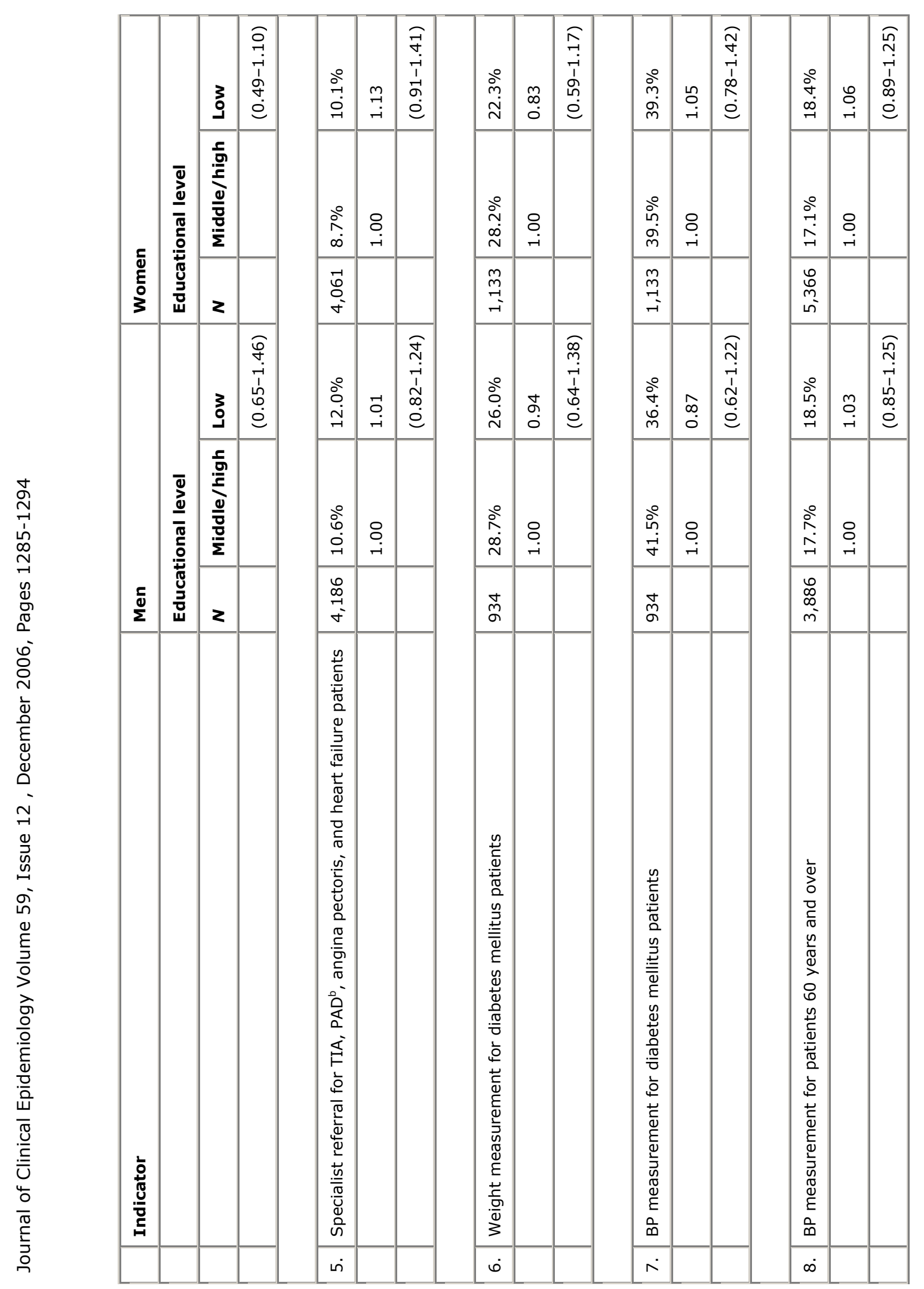


Journal of Clinical Epidemiology Volume 59, Issue 12 , December 2006, Pages 1285-1294

${ }^{a}$ Odds ratios are adjusted for differences across educational level groups in health need, as measured by age, self-perceived health, number of cardiovascular symptoms, specific cardiovascular diseases, diabetes, other comorbidities, and specialist referrals.

${ }^{b}$ Peripheral artery disease. 
Most patients with a hypercholesterolemia diagnosis who were under medication (and therefore in the second-line treatment phase) received a prescription for statins as recommended by the guidelines (Table 4). However, patients with a lower educational level were less likely to receive a prescription for statins. Although we did not have enough power to detect this difference separately for men and women, pooled analyses revealed significant educational level disparities in the prescription for statins for men and women together (OR $=0.62,95 \%$ $\mathrm{CI}=0.42-0.91$ ). This suggests that there were some disparities in the secondline treatment for hypercholesterolemia as recommended by practice guidelines.

\section{Discussion}

Previous research suggests that there are socioeconomic disparities in stroke in most European countries [4], [5] and [8]. In the Netherlands, we found evidence of socioeconomic disparities in stroke incidence in men, but not in women. Among men, socioeconomic disparities in stroke are likely to be due to a higher prevalence of stroke precursors in the lower socioeconomic groups. We observed socioeconomic disparities in the prevalence of hypertension, hypercholesterolemia, diabetes mellitus, angina pectoris, heart failure, and peripheral artery disease. GPs were less likely to comply with guidelines on statin prescriptions for second-line treatment of hypercholesterolemia in patients with a lower socioeconomic status, which can have implications in the prevention of ischemic stroke. However, the quality of care for other precursors of stroke was similar for all socioeconomic groups, or somewhat better for the lower socioeconomic classes. Overall, results emphasize the contribution of socioeconomic disparities in the prevalence of precursors of stroke to disparities in stroke incidence, whereas disparities in the quality of care might play only a minor role.

\subsection{Evaluation of data and study limitations}

We used the most recent data on general practice care in the Netherlands, which comprised a large sample of patients. Nevertheless, some methodological issues should be considered in this study. Stroke or other diseases may have been wrongly diagnosed by the GP. This would have biased our results only if misclassification had occurred differently across educational levels. There is no evidence to suggest that this occurred in our data. Therefore, any bias caused by this problem is likely to have been small. Furthermore, stroke occurrences in patients who went directly to hospital may not have been reported to the GP, which may have led to an underestimation of stroke incidence. However, in the Netherlands, the GP plays a gate-keeping role, so that hospital contacts are generally reported to the GP. Similarly, due the large scale and population registry character of our study, we were not able to determine to what extent the diagnosis of stroke was ascertained by sophisticated diagnostic technique such as computer tomography or magnetic resonance imaging. However, a linkage of data from our study with data on cardiovascular diagnoses from the national hospital registration system in the Netherlands showed high correspondence [26]. Thus, it is unlikely that these problems influenced our results to a great extent.

Data on stroke subtypes were not available in our study. Thus, we used a broad category of stroke comprising both ischemic and hemorrhagic events. We were, therefore, unable to assess the effect of socioeconomic status on different stroke categories. However, previous studies indicate that lower socioeconomic status is consistently associated with higher incidence of ischemic stroke [5], intracerebral [6], and subarachnoid hemorrhage [7], and associations for the three categories seem to be of similar magnitude [5], [6] and [7]. Furthermore, our study was 
primarily focused on assessing disparities in the quality of care for precursors for stroke prevention at general practice. Thus, although it can provide further insight, it is unlikely that including data on stroke subtypes would substantially alter the main conclusions of our study.

Data on prescriptions, referrals, and GP contacts were collected for a period of 12 months. However, information on blood pressure and weight measurements was available for a period of only 6 weeks. Because these measurements may have been carried out in patients at time points outside this period, estimates of their prevalence cannot be interpreted in absolute terms. Nevertheless, we used these estimates solely to assess disparities between socioeconomic groups, rather than overall compliance of GPs with guidelines on hypertension and weight measurements. Thus, odds ratios estimating disparities in these care factors are likely to be comparable to estimates based on a longer data collection period.

Although we obtained a high-response rate in the baseline survey $(76.5 \%)$, the exclusion of nonrespondents may have biased our results to some extent. However, there is evidence that response bias has only a negligible effect on risk estimates of healthcare utilization by background characteristics [27]. To further test the impact of nonresponse, we analyzed differences in the quality of healthcare according to health insurance type, which was available for the total study population. In the Netherlands, health insurance type is a proxy for income, because individuals above an income threshold are required to have a private health insurance. Results for health insurance were identical to results for educational level: Men with public insurance (i.e., below the income threshold) had a higher incidence of stroke than men with private insurance $(O R=1.59$, $95 \%$ CI $=1.23-2.05)$. Similarly, among both men and women, there were large disparities in the prevalence of precursors of stroke, but not in the quality of preventive care. These results suggest that the effect of nonresponse is likely to be small, and further confirm our findings for educational level.

We assessed socioeconomic disparities in eight quality of care indicators. It is possible that there are disparities in other aspects of preventive care not included in this study. However, analyses were also conducted for other indicators regarding blood measurements, prescription for hypertension medication (other than diuretics), referrals of diabetic patients to an ophthalmologist, and ECG examinations among specific groups of patients (results not shown). Similarly, no major socioeconomic disparities were observed in the compliance of GPs to practice guidelines. Nevertheless, further research should assess whether the same pattern applies for other indicators of the quality of preventive care for precursors of stroke.

\subsection{Comparison with previous studies}

The results of this study add to a large body of research showing that a lower socioeconomic status is associated with a higher risk of stroke among men [2], [4], [5], [6], [7], [8] and [9]. Previous studies have observed consistent socioeconomic disparities in stroke mortality in Europe [4] and [8], which are due partly to socioeconomic differences in stroke incidence [5], [6], [7] and [9]. In contrast with findings from previous studies [4], [8] and [28], however, we observed no socioeconomic disparities in stroke incidence for women. This may reflect differences between men and women in the effect of educational level on cardiovascular risk factors. Previous studies have shown that socioeconomic disparities in several cardiovascular risk factors such as smoking and excessive alcohol consumption tend to be larger among men than among women [29], [30] 
and [31], which may have resulted in larger socioeconomic disparities in stroke for men.

Previous studies have also observed socioeconomic disparities in precursors of stroke such as hypertension [10], [28] and [32], diabetes [33], heart failure [34], and hypercholesterolemia [35]. However, studies on socioeconomic differences in care for these conditions are controversial. On the one hand, socioeconomic disparities have been reported in some aspects of care for hypertension [10] and [32], diabetes [10] and [36], and heart failure [34]. In contrast, other studies have observed no socioeconomic disparities in care for these factors [37] and [38]. The results of our study support the view that the quality of cardiovascular preventive care is relatively equal for all socioeconomic classes. This is consistent with previous research suggesting that healthcare plays only a limited role in the explanation of socioeconomic differences in health, which may be due to the equal access to healthcare services enjoyed by the Dutch population [39]. However, this is unlikely to apply to other countries such as the United States, where access to medical care differs between socioeconomic groups [40].

The prescription rate for statins has recently increased among the lower socioeconomic groups [41]. Although patients with a lower socioeconomic status were less likely to be treated with statins during the mid-1990s, these disparities have recently disappeared [41]. However, no research on GPs' compliance with guidelines on statin prescriptions had previously been conducted. This is the first study to report that lower socioeconomic status patients with hypercholesterolemia initiated on cholesterol-lowering medication therapy are less likely to receive statins as choice of medication. This suggests that there still may be socioeconomic disparities in GPs' compliance with guidelines for the prescription of statins for hypercholesterolemia. Although the mechanisms remain unclear, statins have been shown to significantly reduce the risk of ischemic stroke [42]. Thus, these disparities may partly contribute to existing socioeconomic differences in ischemic events [5].

Patients with a low socioeconomic status receive more prescriptions for medication than patients with a high socioeconomic position [43]. Similarly, we found that diuretics were somewhat more often prescribed to hypertension patients with a lower socioeconomic position. However, we did not observe disparities in GPs' compliance with guidelines on the prescription for other medications such as aspirin and ACE-inhibitors. Overall, this suggests that GPs' compliance with guidelines for medication prescriptions is largely similar for patients from different socioeconomic groups.

\subsection{Explanation of results}

In this study, we found no evidence of major disparities in the quality of care provided by GPs to patients from different socioeconomic groups. These findings raise questions regarding the factors of the Dutch primary care system that may have helped to generate equal care for all patients. Firstly, in the Netherlands, healthcare is available to all through health insurance schemes. In our study, the majority of patients with a low educational level were publicly insured, whereby healthcare services were free of charge. This may have contributed to eliminating any financial barriers to access general practice care among the lower socioeconomic groups [11] and [12]. Secondly, quality indicators were constructed on the basis of national guidelines for care in general practice. These are evidence-based guidelines aimed not only at improving the quality of care but also at preventing unnecessary interventions. After adjusting for health need, we observed no differences in the quality of care between patients from different 
socioeconomic groups. Guidelines may have contributed to the provision of equal and standardized diagnostics and care for patients on the basis of health need, thereby leaving limited room for variability in care according to factors such as socioeconomic status. Conversely, there might be disparities in the aspects of care for which evidence is scarce, and therefore no guidelines have been developed. Thus, general practice guidelines may not only contribute to improving the quality of care, but may also promote equal and standardized preventive care across socioeconomic groups.

We found that GPs' compliance with guidelines on the prescription for diuretics as first-line treatment for uncomplicated hypertension [14] and [18] was somewhat higher for patients with a lower socioeconomic status. This suggests that, for some aspects, the quality of care may indeed be better for these patients. These findings are interesting in the light of substantial research showing that lower socioeconomic status is associated with more general practice care consultations and prescriptions [11], [12] and [44]. More frequent contacts with lower socioeconomic groups may provide GPs with more opportunities to comply with preventive guidelines in these patients. For instance, GPs discuss preventive care topics more often with patients from a lower socioeconomic background [45]. Thus, individuals with low socioeconomic status do not only use more general practice care [11], [12] and [44] but might also receive care of a better quality than their higher socioeconomic status counterparts.

Despite this positive scenario, socioeconomic disparities in stroke incidence persist among men. This suggests that general practice care may be merely part of a broader picture, and raises questions about stroke risk factors and precursors that may play a more prominent role in the origin of stroke disparities. Hypertension is a major risk factor for stroke, accounting for up to $60 \%$ of ischemic strokes [20]. The disparities observed in the prevalence of hypertension are likely to contribute to socioeconomic differences in stroke incidence in men [2]. Factors such as hypercholesterolemia and diabetes are also likely to contribute to socioeconomic differences in stroke incidence [2] and [20]. This may be exacerbated by the fact that men with a low socioeconomic status have a higher prevalence of smoking [29], excessive alcohol consumption [46], physical inactivity [47], and overweight [30]. Research indicates that these factors all together explain about half of the socioeconomic differences in stroke incidence [2]. On the other hand, despite observing disparities in stroke precursors, we did not find evidence of socioeconomic disparities in stroke incidence in women. The fact that equal care for stroke precursors was provided for women from different socioeconomic categories may have helped prevent the emergence of disparities in stroke in women.

GPs' compliance with guidelines for the prescription for statins as second-line treatment for hypercholesterolemia was lower in patients with a lower socioeconomic status. This may reflect socioeconomic differences in communication patterns between GPs and patients [12]. Higher educated patients may have a greater awareness of treatment options and therefore be more likely to request certain types of medication. This is consistent with studies showing that patients with a higher socioeconomic position are more likely to be treated with newer or brand-name medications [48]. Further research should explore the explanation of disparities in the management of hypercholesterolemia, and how these differences can contribute to socioeconomic variations in the incidence of ischemic stroke.

Previous studies on healthcare disparities have mostly focused on healthcare utilization [11], [12], [13], [38] and [44]. We developed an innovative approach, 
whereby indicators of quality of care were constructed and GPs' adherence to guidelines assessed. Thus, our study represents one of the first attempts to systematically assess disparities in the quality of preventive care, taking into account patients' health need and general practice variations using multilevel analyses techniques. Nevertheless, the mechanisms through which healthcare can play a role in stroke disparities involve the following [13]. Firstly, socioeconomic status may determine the proportion of individuals receiving high-quality care. Our findings suggest that this mechanism does not play a major role. On the other hand, socioeconomic status may modify the host response to therapeutic interventions, due to differences in factors such as patients' adherence to therapy and susceptibility to the impact of interventions [13]. Thus, even in the absence of disparities in the quality of care by the GP, preventive interventions may still be less effective in the lower socioeconomic groups. Future research should assess whether healthcare may contribute to stroke disparities through this alternative mechanism [13].

Finally, despite the absence of disparities in the quality of care for most stroke precursors in our study, there may still be socioeconomic differences in specialist care relevant for stroke prevention. Although patients with a low socioeconomic status use more general practice care, this is offset by an excess recourse of patients from the high socioeconomic categories to specialist care [11] and [12]. Furthermore, there are socioeconomic differences in treatment and survival after stroke even in countries with universal healthcare systems [5] and [9], which may contribute to socioeconomic disparities in stroke mortality in Europe [4] and [8]. Thus, disparities in the quality of care for stroke may exist at more specialized levels of the healthcare system.

\subsection{Implications}

Universal access to general practice care and the implementation of general practice guidelines are likely to have promoted the provision of equal and standardized care for stroke precursors across socioeconomic groups. However, this has not been sufficient to eliminate socioeconomic disparities in stroke occurrence. Therefore, reducing the prevalence of stroke precursors such as hypertension, hypercholesterolemia, and diabetes in the lower socioeconomic groups is still necessary. Interventions focused on the primary prevention of factors such as an unhealthy diet, smoking, and obesity may play a major role in reducing stroke disparities. Furthermore, improvements in GPs' compliance with guidelines for the prescription for statins as second-line treatment for hypercholesterolemia may contribute to reduce disparities in ischemic stroke incidence. Overall, reducing disparities in the primary occurrence of stroke precursors may be more important than reducing disparities in subsequent preventive care. GPs may thus play a key role in reducing disparities in stroke through primary prevention of risk factors for stroke.

\section{Acknowledgments}

The central source of funding of the Dutch national survey of general practice was the Dutch Ministry of Health, Welfare and Sport. Data were collected by the Netherlands Institute for Health Services Research (NIVEL) and the National Institute of Public Health and the Environment (RIVM). During the preparation phase of this manuscript, F.J. Van Lenthe was supported by a grant from the Netherlands Organization for Scientific Research (NWO grant number 904-66104). 


\section{References}

[1] C.J. Murray and A.D. Lopez, Mortality by cause for eight regions of the world: global burden of disease study, Lancet 349 (1997), pp. 1269-1276.

[2] C.L. Hart, D.J. Hole and G.D. Smith, The contribution of risk factors to stroke differentials, by socioeconomic position in adulthood: the Renfrew/Paisley Study, Am J Public Health 90 (2000), pp. $1788-1791$.

[3] C.L. Chang, M.G. Marmot, T.M. Farley and N.R. Poulter, The influence of economic development on the association between education and the risk of acute myocardial infarction and stroke, J Clin Epidemiol 55 (2002), pp. 741-747.

[4] M. Avendano, A.E. Kunst, F. van Lenthe, V. Bos, G. Costa and T. Valkonen et al., Trends in socioeconomic disparities in stroke mortality in six European countries between 1981-1985 and 19911995, Am J Epidemiol 161 (2005), pp. 52-61.

[5] D. Jakovljevic, C. Sarti, J. Sivenius, J. Torppa, M. Mahonen and P. Immonen-Raiha et al., Socioeconomic status and ischemic stroke: The FINMONICA stroke register, Stroke 32 (2001), pp. 1492-1498.

[6] D. Jakovljevic, C. Sarti, J. Sivenius, J. Torppa, M. Mahonen and P. Immonen-Raiha et al., Socioeconomic differences in the incidence, mortality and prognosis of intracerebral hemorrhage in Finnish adult population. The FINMONICA Stroke Register, Neuroepidemiology 20 (2001), pp. 85-90.

[7] D. Jakovljevic, J. Sivenius, C. Sarti, J. Torppa, M. Mahonen and P. Immonen-Raiha et al., Socioeconomic inequalities in the incidence, mortality and prognosis of subarachnoid hemorrhage: the FINMONICA Stroke Register, Cerebrovasc Dis 12 (2001), pp. 7-13.

[8] M. Avendano, A.E. Kunst, M. Huisman, F. van Lenthe, M. Bopp and C. Borrell et al., Educational level and stroke mortality: a comparison of 10 European populations during the 1990 s, Stroke 35 (2004), pp. 432-437.

[9] M. Peltonen, M. Rosen, V. Lundberg and K. Asplund, Social patterning of myocardial infarction and stroke in Sweden: incidence and survival, Am J Epidemiol 151 (2000), pp. 283-292.

[10] J.S. De Koning, N. Klazinga, P.J. Koudstaal, A. Prins, G.J. Borsboom and A. Peeters et al., Deprivation and systematic stroke prevention in general practice: an audit among general practitioners in the Rotterdam region, The Netherlands, Eur J Public Health 13 (2003), pp. 340-346.

[11] E. Van Doorslaer, X. Koolman and A.M. Jones, Explaining income-related inequalities in doctor utilisation in Europe, Health Econ 13 (2004), pp. 629-647.

[12] J.B. Van der Meer, J. van den Bos and J.P. Mackenbach, Socioeconomic differences in the utilization of health services in a Dutch population: the contribution of health status, Health Policy 37 (1996), pp. 1-18 (Amsterdam, Netherlands).

[13] G.M. Anderson, S.E. Bronskill, C.A. Mustard, A. Culyer, D.A. Alter and D.G. Manuel, Both clinical epidemiology and population health perspectives can define the role of health care in reducing health disparities, J Clin Epidemiol 58 (2005), pp. 757-762.

[14] Nederlands Huisarten Genootschap, NHG-Standaarden, Nederlands Huisarten Genootschap, Utrecht (2004) Updated December 22, 2004. Available athttp://nhg.artsennet.nl/content/resources//AMGATE_6059_104_TICH_R1195991373945248// Accessed May 15, 2004.

[15] M.K. Kapral, H. Wang, M. Mamdani and J.V. Tu, Effect of socioeconomic status on treatment and mortality after stroke, Stroke 33 (2002), pp. 268-273.

[16] G.P. Westert, F.G. Schellevis, D.H. de Bakker, P.P. Groenewegen, J.M. Bensing and J. van der Zee, Monitoring health inequalities through general practice: the Second Dutch National Survey of General Practice, Eur J Public Health 15 (2005), pp. 59-65. 
[17] T. Valkonen, Problems in the measurement and international comparisons of socio-economic differences in mortality, Soc Sci Med 36 (1993), pp. 409-418.

[18] J. Braspenning, E.G. Schellevis and R.P.T.M. Grol, Tweede Nationale Studie Naar Ziekten en Verrichtingen in de Huisartspraktijk: Kwaliteit Huisartsenzorg Belicht, Nivel Instituut, Utrecht (2004).

[19] S.M. Campbell, J. Braspenning, A. Hutchinson and M.N. Marshall, Research methods used in developing and applying quality indicators in primary care, BMJ 326 (2003), pp. 816-819.

[20] L.B. Goldstein, R. Adams, K. Becker, C.D. Furberg, P.B. Gorelick and G. Hademenos et al., Primary prevention of ischemic stroke: a statement for health care professionals from the Stroke Council of the American Heart Association, Circulation 103 (2001), pp. 163-182.

[21] H. Lamberts and M. Wood, ICPC: international classification of primary care, Oxford University Press, New York (1987).

[22] World Health Organization, Anatomic, Therapeutic, Chemical Classification (ATC), WHO, Geneva (1999).

[23] Centraal Bureau voor de Statistiek, Statistisch Jaarboek 2004, Centraal Bureau voor de Statistiek, Heerlen (2004).

[24] E.L. Korn, B.I. Graubard and D. Midthune, Time-to-event analysis of longitudinal follow-up of a survey: choice of the time-scale, Am J Epidemiol 145 (1997), pp. 72-80.

[25] T.M. Therneau and P.M. Grambsch, Multiple events per subject. In: T.M. Therneau and P.M. Grambsch, Editors, Modeling survival data: extending the Cox Model, Springer, New York (2000), pp. 169-230 (Chapter 8).

[26] J.N. Struijs, C.A. Baan, L.C.J. Slobbe, M. Droomers and G.P. Westert, Koppeling van anonieme huisartsgegevens aan ziekenhuisregistraties, RIVM, Bilthoven (2004).

[27] S.A. Reijneveld and K. Stronks, The impact of response bias on estimates of health care utilization in a metropolitan area: the use of administrative data, Int J Epidemiol 28 (1999), pp. 11341140.

[28] C.T. Van Rossum, H. van de Mheen, M.M. Breteler, D.E. Grobbee and J.P. Mackenbach, Socioeconomic differences in stroke among Dutch elderly women: the Rotterdam Study, Stroke 30 (1999), pp. 357-362.

[29] A.E. Cavelaars, A.E. Kunst, J.J. Geurts, R. Crialesi, L. Grotvedt and U. Helmert et al., Educational differences in smoking: international comparison, BMJ 320 (2000), pp. 1102-1107.

[30] A. Cavelaars, A. Kunst and J.P. Mackenbach, Socio-economic differences in risk factors for morbidity and mortality in the European Community: an international comparison, J Health Psychol 2 (1997), pp. 353-372.

[31] M. Droomers, C.T. Schrijvers and J.P. Mackenbach, Educational differences in starting excessive alcohol consumption: explanations from the longitudinal GLOBE study, Soc Sci Med 58 (2004), pp. 2023-2033.

[32] R. de Gaudemaris, T. Lang, G. Chatellier, L. Larabi, V. Lauwers-Cances and A. Maitre et al., Socioeconomic inequalities in hypertension prevalence and care: the IHPAF Study, Hypertension 39 (2002), pp. 1119-1125.

[33] E.E. Agardh, A. Ahlbom, T. Andersson, S. Efendic, V. Grill and J. Hallqvist et al., Explanations of socioeconomic differences in excess risk of type 2 diabetes in Swedish men and women, Diabetes Care 27 (2004), pp. 716-721.

[34] F.A. McAlister, N.F. Murphy, C.R. Simpson, S. Stewart, K. MacIntyre and M. Kirkpatrick et al., Influence of socioeconomic deprivation on the primary care burden and treatment of patients with a diagnosis of heart failure in general practice in Scotland: population based study, BMJ 328 (2004), p. 1110 
[35] N. Hoeymans, H.A. Smit, H. Verkleij and D. Kromhout, Cardiovascular risk factors in relation to educational level in 36000 men and women in The Netherlands, Eur Heart J 17 (1996), pp. 518-525.

[36] J. Hippisley-Cox, S. O'Hanlon and C. Coupland, Association of deprivation, ethnicity, and sex with quality indicators for diabetes: population based survey of 53,000 patients in primary care, BMJ 329 (2004), pp. 1267-1269.

[37] S. Shah and D.G. Cook, Inequalities in the treatment and control of hypertension: age, social isolation and lifestyle are more important than economic circumstances, J Hypertens 19 (2001), pp. 1333-1340.

[38] J.B. Van der Meer and J.P. Mackenbach, The care and course of diabetes: differences according to level of education, Health Policy 46 (1999), pp. 127-141.

[39] G.A. van den Bos, J.P. Smits, G.P. Westert and A. van Straten, Socioeconomic variations in the course of stroke: unequal health outcomes, equal care?, J Epidemiol Community Health 56 (2002), pp. 943-948.

[40] A.Y. Chen and J.J. Escarce, Quantifying income-related inequality in health care delivery in the United States, Med Care 42 (2004), pp. 38-47.

[41] C. packham, J. pearson, J. Robinson and D. Gray, Use of statins in general practices, 1996-1998: cross sectional study, BMJ 320 (2000), pp. 1583-1584.

[42] P. Amarenco, J. Labreuche, P. Lavallee and P.J. Touboul, Statins in stroke prevention and carotid atherosclerosis: systematic review and up-to-date meta-analysis, Stroke 35 (2004), pp. 2902-2909.

[43] E. Odubanjo, K. Bennett and J. Feely, Influence of socioeconomic status on the quality of prescribing in the elderly-a population based study, Br J Clin Pharmacol 58 (2004), pp. 496-502.

[44] J.B. Van der Meer and J.P. Mackenbach, Low education, high GP consultation rates: the effect of psychosocial factors, J Psychosom Res 44 (1998), pp. 587-597.

[45] J.H. Wiggers and R. Sanson-Fisher, Practitioner provision of preventive care in general practice consultations: association with patient educational and occupational status, Soc Sci Med 44 (1997), pp. 137-146.

[46] M. Droomers, C.T. Schrijvers, K. Stronks, D. van de Mheen and J.P. Mackenbach, Educational differences in excessive alcohol consumption: the role of psychosocial and material stressors, Prev Med 29 (1999), pp. 1-10.

[47] M. Droomers, C.T. Schrijvers, H. van de Mheen and J.P. Mackenbach, Educational differences in leisure-time physical inactivity: a descriptive and explanatory study, Soc Sci Med 47 (1998), pp. 1665-1676.

[48] M.M. Mamdani, K. Tu, P.C. Austin and D.A. Alter, Influence of socioeconomic status on drug selection for the elderly in Canada, Ann Pharmacother 36 (2002), pp. 804-808. 